\title{
JOINT ENCODING OF THE DEPTH IMAGE BASED REPRESENTATION USING SHAPE-ADAPTIVE WAVELETS
}

\author{
Matthieu Maitre and Minh N. Do \\ Department of Electrical and Computer Engineering \\ University of Illinois at Urbana-Champaign \\ \{maitre,minhdo\}@uiuc.edu
}

\begin{abstract}
We present a novel codec of depth-image-based representations for free-viewpoint 3D-TV. The proposed codec relies on a shape-adaptive wavelet transform and an explicit representation of the locations of major depth edges. Unlike classical wavelet transforms, the shape-adaptive transform generates small wavelet coefficients along depth edges, which greatly reduces the data entropy. The codec also shares the edge information between the depth map and the image to reduce their correlation. The wavelet transform is implemented by shape-adaptive lifting, which enables fast computations and perfect reconstruction. Experimental results on real data confirm the superiority of the proposed codec, with PSNR gains of up to $5.46 \mathrm{~dB}$ on the depth map and up to $0.19 \mathrm{~dB}$ on the image compared to standard wavelet codecs.
\end{abstract}

Index Terms - 3D-TV, shape-adaptive wavelet transform, lifting, boundary wavelets, edge coding, joint coding

\section{INTRODUCTION}

Free-viewpoint three-dimensional television (3D-TV) provides an enhanced viewing experience in which users are able to perceive the third spatial dimension and are free to move inside the 3D video [1]. Three-dimensional television poses new technological challenges, which include recording, encoding and displaying $3 \mathrm{D}$ videos. At the core of these challenges lies the massive amount of data required to represent the set of all possible views, or at least a realistic approximation of them.

The Depth-Image-Based Representation (DIBR) has recently emerged as an effective approach [2], which allows both compact data representation and realistic view synthesis. As shown in Figure 1, the DIBR is made of pairs of images and depth maps, each of which provide a local approximation of the plenoptic function. At the decoder, arbitrary views are synthesized from the DIBR using image-based rendering [3].

Each pair of image and depth map can be seen as a four channel image with one channel for luma, two for chroma and one for depth. Therefore, classical image and video codecs like MPEG-2, H.264/AVC and JPEG2000 only need minor modifications to be able to handle DIBRs [1,2]. This approach however fails to take into consideration the fact that images and depth maps exhibit widely different statistics, which make classical transforms like the Discrete Wavelet Transform (DWT) or the Discrete Cosine Transform (DCT) ill-suited to encode DIBRs.

In [4], it was shown that a representation based on platelets, which assumes piecewise planar areas separated by piecewise linear edges, could lead to major Rate-Distortion (RD) gains. However, the

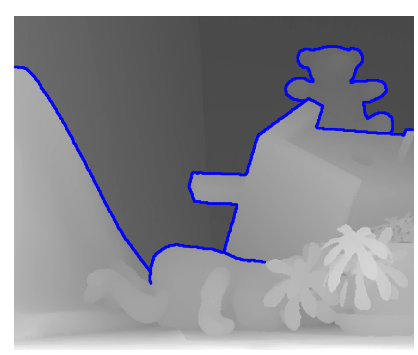

(a) Depth map and edges

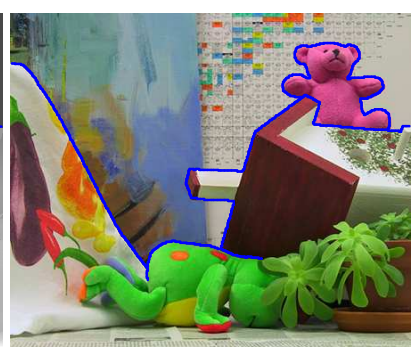

(b) Image and edges
Fig. 1. Input data of the proposed DIBR codec: shared edges superimposed over a depth map (a) and an image (b).

practical use of platelets is limited by the computational cost of encoding. Unlike standard image codecs which rely on fast transforms (such as DCT or DWT), quantizers and entropy coders to encode the data [5], platelets require the encoder to solve a complex RD optimization problem.

Moreover, both platelet-based codecs and standard image codecs ignore another source of data redundancy: the correlation between depth edges and image edges. Indeed, the 3D scenes are usually made of objects with well-defined surfaces, which, by projection onto the camera image plane, create edges at the same locations in the depth map and the image.

In this paper, we propose a codec which takes into account both sources of redundancies. It encodes the locations of the major depth edges explicitly and treats the regions they separate as independent during the wavelet transform by using an extension of the ShapeAdaptive Discrete Wavelet Transform (SA-DWT) [6]. The proposed SA-DWT generates small wavelet coefficients both in smooth regions and along the encoded edges. It is efficiently computed using lifting [5], a procedure which is fast, in place, simple to implement and trivially invertible. Moreover, the explicit edge coding allows the codec to share the edge information between the depth map and the image, which reduces their joint redundancy.

Thus the proposed codec amounts to a simple modification of a scheme that independently codes depth and image using waveletbased codecs. As a result, we can benefit from the large body of existing work on wavelet-based codecs. However, as we shall see, this modification leads to significant gains, up to $5.46 \mathrm{~dB}$.

The remainder of the article is organized as follows. An overview of the proposed coded is presented in Section 2. A detailed description of its components is given in Sections 3, 4 and 5, which consider respectively the SA-DWT, the handling of edges during lifting and edge coding. Finally, Section 6 presents experimental results. 


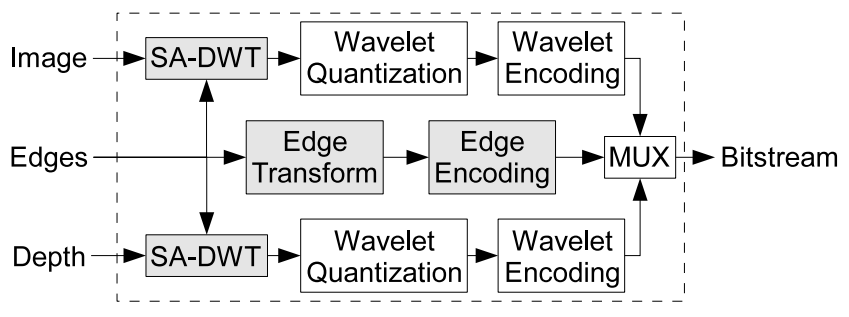

Fig. 2. Overview of the proposed encoder. It relies on a SA-DWT and an edge coder (gray boxes) to reduce data correlations, both intra and inter the image and the depth map.

\section{PROPOSED CODEC}

As shown in Figure 2, the proposed DIBR encoder takes three signals as input, which represent respectively the depth map, the image and the edges. The DIBR encoder is made of two wavelet encoders [5], one processing the depth map and the other the image. Like standard wavelet encoders, each is made of a wavelet transform followed by quantization and entropy coding. The DIBR decoder simply inverses each of these steps in reverse order.

The major novelty of the proposed encoder lies in the introduction of a transform by SA-DWT for both the image and the depth map. SA-DWT requires an explicitly representation of edges, which leads to the introduction of a lossless edge encoder made of an edge transform and entropy coding. The explicit edge representation has the advantage that it can be shared by the both the image and depth SA-DWTs, which leads to bitrate savings.

The DIBR encoder generates three bitstreams (image, depth, edge), which are eventually concatenated by a multiplexer (MUX).

\section{SHAPE-ADAPTIVE WAVELET TRANSFORM}

SA-DWT [6] relies on the notion of image object. An image object is made of a binary mask, which indicates which pixels are inside the object, and an image. The image values at outside pixels are assumed to be missing. The object is transformed by an adequate downsampling of the mask and a DWT of the image, extrapolating the missing values around the object boundary by symmetric extension. The 2D SA-DWT is implemented as separable 1D SA-DWTs and the process is iterated on the low-pass band to obtain a multiresolution transform. SA-DWT has the advantage of avoiding creating large wavelet coefficients around the object boundary by treating the inside and outside areas as statistically independent.

This is this idea of statistically independent areas that we shall use to efficiently code the DIBR. In our case, however, there is no single object. Instead, the DIBR is made of multiple superimposed objects. This issue is overcome by replacing the binary mask by a binary edge map and by treating the areas on opposite sides of the edges as statistically independent.

Figure 3 presents an example of signal processed by the proposed SA-DWT. The SA-DWT clearly creates much less non-zero wavelet coefficients around edges than the standard DWT, which leads to bitrate savings. SA-DWT has the disadvantage of requiring a bitrate overhead to code the edge location. However, experiments show that this overhead is more than compensated by the savings.

We rely on lifting [5] to implement the 1D SA-DWT. First, we present the standard lifting and then describe the modifications making it shape adaptive. The lifting splits the samples at odd and even

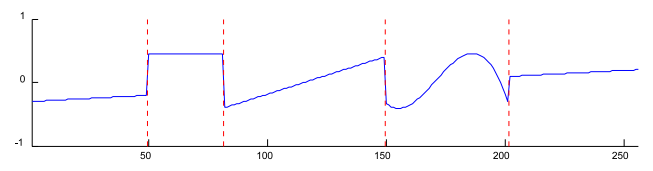

(a) Signal made of constant, linear and cubic pieces. The dashed red lines indicate edges.

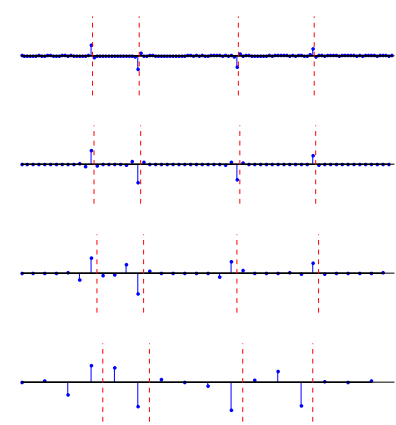

(b) High-pass bands of a standard 9/7 DWT with symmetric extension

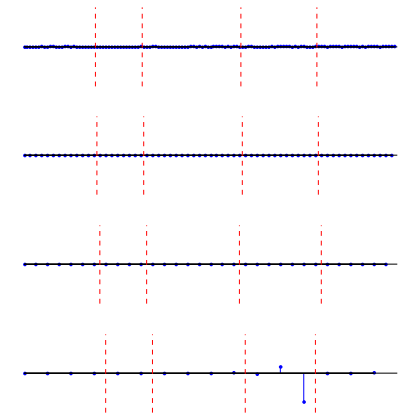

(c) High-pass bands of a 9/7 SADWT with cubic extension
Fig. 3. Comparison of standard and shape adaptive DWTs. In the latter case, all but the coarsest high-pass band are zero.

locations into two cosets and modify them alternatively using a series of "steps."

By convention, lifting begins by modifying the odd coset by a so-called "predict" step, which transforms a signal $x$ into a signal $y$ such that

$$
\left\{\begin{aligned}
y_{2 t} & =x_{2 t}, \\
y_{2 t+1} & =x_{2 t+1}+\lambda_{2 k}\left(x_{2 t}+x_{2 t+2}\right),
\end{aligned}\right.
$$

where $t$ denotes the sample locations, $2 k$ the step number and $\lambda_{2 k}$ a weight. The even coset is modified by a so-called "update" step, which transforms a signal $x$ into a signal $y$ such that

$$
\left\{\begin{aligned}
y_{2 t} & =x_{2 t}+\lambda_{2 k+1}\left(x_{2 t-1}+x_{2 t+1}\right), \\
y_{2 t+1} & =x_{2 t+1} .
\end{aligned}\right.
$$

Figure 4 shows a graphical example of these lifting steps. After the final update step, the odd coset contains the high-pass coefficients and the even coset the low-pass ones.

Standard lifting is made shape adaptive by modifying (1) and (2) at locations where they would perform a weighted addition of two samples $x_{t}$ and $x_{t^{\prime}}$ separated by an edge. In that case, the sample $x_{t^{\prime}}$ which is not on the same side of the edge as the sample $y_{t}$ being computed is considered missing and is extrapolated from samples on the same side as $y_{t}$.

\section{LIFTING EDGE HANDLING}

Our goal is to design extrapolation methods which lead to null highpass coefficients around edges. The symmetric extension [6] ensures the continuity of the signal across the edge but not of its higher order derivatives. Therefore, even wavelets which zero out high-order polynomials are not able to zero them out near edges. The extrapolation design we now present is able to overcome this limitation.

In the following, we only consider extrapolation on the right side of edges, as shown in Figure 4. Left-side extrapolation follows by 


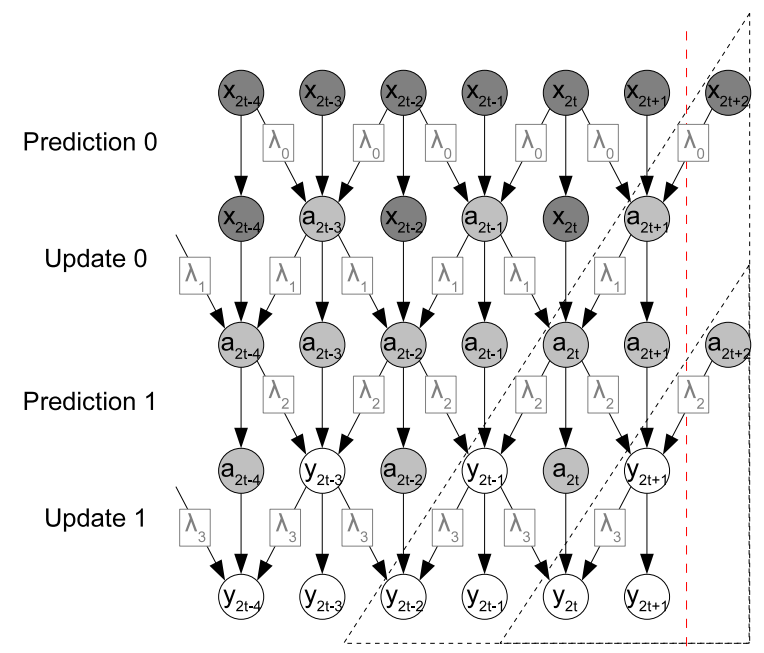

Fig. 4. The four lifting steps associated with a 9/7 wavelet, which transform the signal $x$ first into $a$ and then into $y$. The values $x_{2 t+2}$ and $a_{2 t+2}$ on the other side of the edge (dashed red line) are extrapolated. They have dependencies with the values inside the two dashed triangles.

symmetry. Let us assume that there is an edge at $T+1 / 2$ and that left of this edge the signal is polynomial of degree $L$, that is,

$$
x_{t}=\sum_{k=0}^{L} \alpha_{k} t^{k}, \forall t \leq T .
$$

In order for lifting to be performed, some of the samples on the right side of the edge need to be estimated. In Figure 4, where $T=2 t+1$, these samples are $x_{2 t+2}$ and $a_{2 t+2}$. We choose to extrapolate them using a weighted sum of the samples on the left of the edge, using an equation of the form

$$
z_{T+1}=\sum_{k=0}^{L} \mu_{k} z_{T-1-2 k}
$$

where $z$ denotes the signal and $\mu$ the vector of unknown weights. The extrapolation only relies on samples from the same coset to maintain invertibility and in-place computation.

If the wavelet zeroes out polynomials of degree $L$, all high-pass coefficients left of the edge should be zero. Among all these equations, only those near the edge depend on $\mu$. In Figure 4, this corresponds to $y_{2 t-1}$ and $y_{2 t+1}$. We write them as functions of $\mu, \lambda$ and $T$ using (1), (2), (3) and (4). Setting them to zero gives a system of polynomials in $T$. We want the solution to be invariant to even shifts of the edge, which means that the polynomials must be identically zero. Therefore all the polynomial coefficients are zero, which gives rise to $L$ new equations per polynomial. The unknown weights $\mu$ are obtained by solving this system of equations.

Writing and solving the equations may be quite tedious but can be easily done by mathematical software and the solutions are particularly simple. In the case of 5/3 wavelets, which zeroes out linear polynomials, an extrapolation with $L=0$ gives the standard symmetric extension with $\mu=1$ and an extrapolation with $L=1$ gives a linear extension with $\mu=\left[\begin{array}{ll}2 & -1\end{array}\right]$. In the case of the $9 / 7$, which zeroes out cubic polynomials, both of these extrapolations hold true and an extrapolation with $L=3$ gives a cubic extension

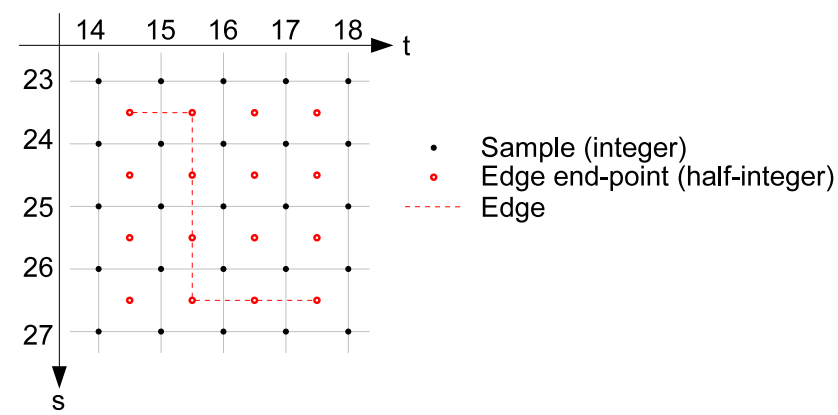

Fig. 5. Example of the dual lattices of samples and edges. Each edge indicate the statistical independence of the two half rows or half columns of samples it separates.

with $\mu=\left[\begin{array}{llll}4 & -6 & 4 & -1\end{array}\right]$. The effectiveness of this latter extrapolation is demonstrated in Figure 3.

The proposed method fails in two cases. First, when the edge location is of the form $2 t+1 / 2$, the unknown high-pass coefficient $y_{2 t+1}$ has a dependency with the low-pass coefficient $y_{2 t}$ but not with any high-pass coefficient on the left of the edge. There is therefore no equation available to solve its extrapolation. However, we can assume that the polynomial if perfectly fitted on the right side of the edge, which means that $y_{2 t+1}$ is zero. The extrapolation also fails when there is an insufficient number of samples between two edges. In that case, the order $L$ of the extrapolation is reduced.

\section{EDGE REPRESENTATION AND CODING}

The SA-DWT requires an explicit knowledge of the edge locations. In 1D, these edges are located at half-integer locations between samples. In 2D, they are located between samples along rows and columns, which gives rise to an edge lattice dual to the sample lattice, as shown in Figure 5. Edges are either horizontal, splitting columns, or vertical, splitting rows. We represent them by two binary edge maps, denoted respectively $e_{s+1 / 2, t}^{(h)}$ and $e_{s, t+1 / 2}^{(v)}$ where $s$ and $t$ denote integer spatial locations.

Moreover, the SA-DWT is a multi-resolution transform where each low-pass band must be associated with a pair of edge maps. Let use denote by $x_{s, t, j}$ the samples at resolution level $j$ and by $e_{s+1 / 2, t, j}^{(h)}$ and $e_{s, t+1 / 2, j}^{(v)}$ the associated depth maps. The pyramid of edge maps is obtained by iterative downsampling using the equations

$$
\left\{\begin{array}{l}
e_{s+1 / 2, t, j}^{(h)}=\max \left(e_{2 s+1 / 2,2 t, j-1}^{(h)}, e_{2 s+1+1 / 2,2 t, j-1}^{(h)}\right) \\
e_{s, t+1 / 2, j}^{(v)}=\max \left(e_{2 s, 2 t+1 / 2, j-1}^{(v)}, e_{2 s, 2 t+1+1 / 2, j-1}^{(v)}\right)
\end{array}\right.
$$

The edge maps at the finest resolution $j=0$ are encoded using a differential Freeman chain code inspired from [7].

\section{EXPERIMENTAL RESULTS}

We present experimental results on the Teddy set, shown in Figure 1. The DIBR is $375 \times 450$ in size with intensities in the range [0,255] and depths in [0,53]. For simplicity, only the luma channel of the image is considered. Missing values in the depth maps have been interpolated using in-painting. Edges have been obtained in a semiautomatic way by applying a Canny edge detector to the depth map and letting the user choose which edge chains to keep. 


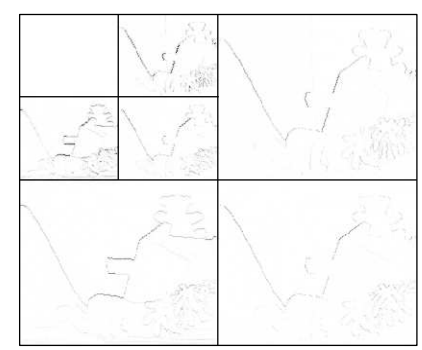

(a) Std $9 / 7$ sym

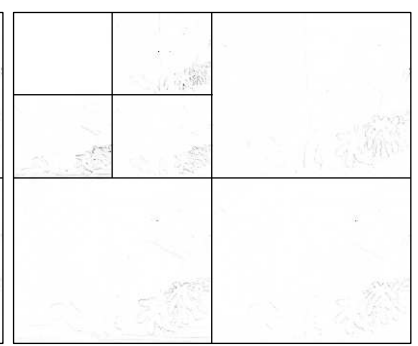

(b) SA9/7lin
Fig. 6. Absolute values of the high-pass coefficients of the depth map using standard and shape-adaptive wavelets. The latter provides a much sparser decomposition.

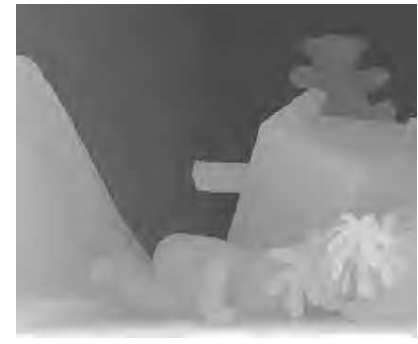

(a) Std9/7sym - 36.21dB

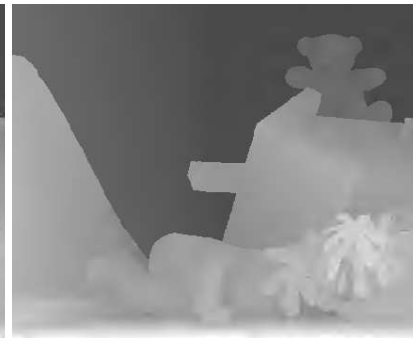

(b) SA9/7lin - 39.86dB
Fig. 7. Reconstruction of the depth map at $0.04 \mathrm{bpp}$ using standard and shape-adaptive wavelets. The latter gives sharp edges free of Gibbs artifacts.

We compare the performances of two codecs: one based on the DWT and the other on the proposed SA-DWT with explicit edge coding. Both codecs perform a five-level decomposition and rely on the same quantizer and entropy coder, provided by the SPIHT implementation of the QccPack library. They also rely on the same 9/7 wavelet for the transform, which is the main wavelet in JPEG2000 [5]. Following [8], both codecs allocate $20 \%$ of the bitrate to the depth.

The two codecs differ on their handling of edges: the DWTbased codec uses the standard 9/7 wavelet with symmetric extension, denoted "std9/7sym", while the SA-DWT-based codec uses the shape-adaptive 9/7 wavelet with linear extension, denoted "SA9/7lin," for the depth map and the shape-adaptive 9/7 wavelet with symmetric extension, denoted "SA9/7sym," for the image. The SA-DWTbased codec also includes an edge codec, as shown in Figure 2. The experiments are based on the edge map shown in Figure 1, which has a bitrate overhead of $0.015 \mathrm{bpp}$.

Figure 6 shows the wavelet coefficients of the depth map obtained using std9/7sym and SA9/7lin. The standard transform exhibits large values along edges, which are absent from the shapeadaptive transform. The entropy of the latter is therefore much reduced.

Figure 7 shows the reconstructed depth map at 0.04bpp using the standard and shape-adaptive codecs. Even at low bitrates, the latter reconstructs sharp edges and avoids Gibbs artifact along edges. Moreover, the sparser nature of the SA-DWT coefficients means that the shape-adaptive codec is able to spend more bits outside edge areas, which leads for instance to a slightly better reconstruction of the bottom right of the depth map.

Figure 8(a) compares the RD performances of the two codecs for the depth map. The bitrate consists in the wavelet coefficients, along

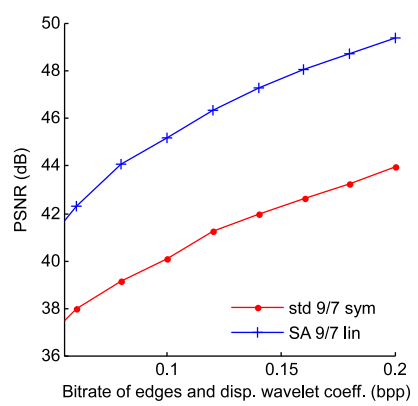

(a) Depth map

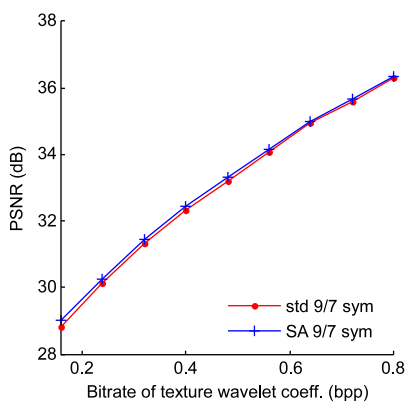

(b) Image
Fig. 8. Rate-distortion performances of standard and shape-adaptive wavelets. The latter gives PSNR gains of up to $5.46 \mathrm{~dB}$ on the depth map and $0.19 \mathrm{~dB}$ on the image.

with the edges in the shape-adaptive case. The figure shows that the edge overhead is more than compensated by the reduced entropy of the wavelet coefficients. This leads to PSNR gains over the whole bitrate range, achieving up to $5.46 \mathrm{~dB}$.

Figure 8(b) compares the RD performances of the two codecs for the image. The bitrate is made of the wavelet coefficients in both cases, the edge overhead having been accounted for in the depth bitrate. The figure shows another PSNR gains over the whole bitrate range, achieving up to $0.19 \mathrm{~dB}$.

\section{CONCLUSION}

We have presented a novel codec of DIBRs for free-viewpoint 3DTV. By replacing the DWT of classical image codecs by a SA-DWT and adding an edge encoder, we have been able to obtain significant PSNR gains, achieving up to $5.46 \mathrm{~dB}$. Future work shall consider the automatic extraction of edges based on RD considerations.

\section{REFERENCES}

[1] C. Fehn, R. Barre, and R. S. Pastoor, "Interactive 3-D TV concepts and key technologies," Proc. of the IEEE, vol. 94, no. 3, pp. 524-538, 2006.

[2] A. Smolic and P. Kauff, "Interactive 3-D video representation and coding," Proc. of the IEEE, vol. 93, no. 1, pp. 98-110, 2005.

[3] Heung-Yeung Shum, Shing-Chow Chan, and Sing Bing Kang, Image-Based Rendering, Springer-Verlag, 2007.

[4] Y. Morvan, D. Farin, and P. H. N. de With, "Depth-image compression based on an R-D optimized quadtree decomposition for the transmission of multiview images," in Proc. ICIP, 2007.

[5] D. Taubman and M. Marcellin, JPEG2000: Image Compression Fundamentals, Standards and Practice, Springer-Verlag, 2001.

[6] S. Li and W. Li, "Shape-adaptive discrete wavelet transforms for arbitrarily shapedvisual object coding," IEEE Trans. on Circuits and Sys. for Video Tech., vol. 10, pp. 725-743, 2000.

[7] Y. K. Liua and B. alik, "An efficient chain code with huffman coding," Pattern Recog., vol. 38, pp. 553-557, 2005.

[8] M. Maitre, Y. Shinagawa, and M. N. Do, "Wavelet-based joint estimation and encoding of depth-image-based representations for free-viewpoint rendering," IEEE Trans. on Im. Proc. (submitted), 2008. 